\title{
Eight years after IYA2009: a successful project of Outreach and Astronomy Education on selected countryside towns in Mexico
}

\author{
Hector Bravo-Alfaro ${ }^{1, \star}$, Cesar A. Caretta ${ }^{\star \star}$, Felipe Macias Gloria ${ }^{2, \star \star \star}$, Elcia M. S. Brito ${ }^{3, \star \star \star \star}$, \\ Patricia Campos Rodríguez ${ }^{2, \dagger}$, Juan Pablo Torres-Papaqui ${ }^{1, \star}$, and Eloy Juarez Sandoval ${ }^{4, \S}$ \\ ${ }^{1}$ Departamento de Astronomía, Universidad de Guanajuato. Apdo. Postal 144, Guanajuato 36000. México. \\ ${ }^{2}$ División de Derecho, Política y Gobierno, Universidad de Guanajuato. Lascurain de Retana 5, Guanajuato \\ 36000. México. \\ ${ }^{3}$ Departamento de Ingeniería Geomática e Hidráulica. Universidad de Guanajuato. Lascurain de Retana 5, \\ Guanajuato 36000. México. \\ ${ }^{4}$ Rectoría General, Universidad de Guanajuato. Lascurain de Retana 5, Guanajuato 36000. México.
}

\begin{abstract}
We present the main results of a multidisciplinary project on education and outreach in Guanajuato (Mexico). This collaboration was initiated in the International Year of Astronomy (IYA09, IAU-UNESCO, 2009) and is led by a team including astronomers, sociologists and environment biologists. The Astronomy activities had their origin at the Public Observatory of Universidad de Guanajuato, where an outreach program was dedicated to young students, teachers and the general public. Thanks to the many activities linked to IYA09, the astronomers joined with a group of sociologists working on the development of rural zones of the Mexican State of Guanajuato. This region undergoes a high rate of migration towards the USA. Working together, our goals were enlarged, having important results on different aspects like social development, environment recovering and education. Astronomy plays a central role within this project, mainly through activities like stargazing, science games for young public, Astronomy courses for teachers, and talks for a wide audience.
\end{abstract}

\section{Introduction: the Roof-top Astronomical Observatory}

The Astronomy Department of Universidad de Guanajuato (UG), was founded in 1995. Since those years, and in parallel with scientific and teaching tasks, the group of professional astronomers developed a series of outreach activities in the city of Guanajuato, the capital of the State of Guanajuato (located in the center of Mexico). At the beginning of the 2000's, the facilities of a former astronomical observatory, located on the roof top of the main building of UG (see Fig. 1), was placed

\footnotetext{
^e-mail: hector@astro.ugto.mx

$\star \star$ e-mail: caretta@astro.ugto.mx

$\star \star \star$ e-mail: chima19933@hotmail.com

$\star \star \star \star$ e-mail: emsbrito@gmail.com

†e-mail: chima19933@hotmail.com

‡e-mail: papaqui@astro.ugto.mx

§e-mail: ejuarez@ugto.mx
} 
under management of the astronomers. One of the coauthors of this paper (HBA) led the works of refurbishment and the acquisition of modern (amateurs) telescopes, as well as computing and audio visual equipment for a room with capacity for 50 people, installed at the same terrace. The many activities organized at the Observatory quickly became well known beyond the city of Guanajuato [1].

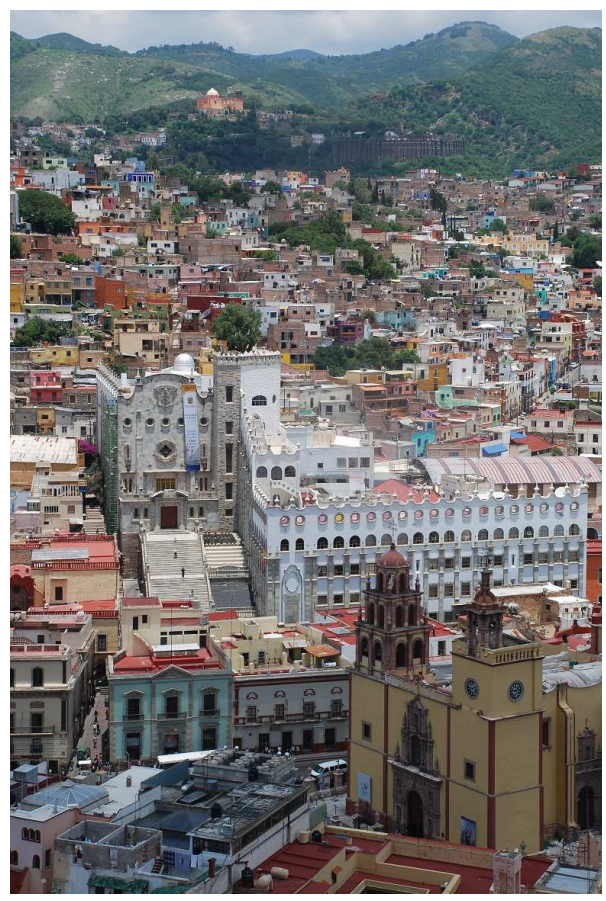

Figure 1. View of the town of Guanajuato. At the center, the main building of Universidad de Guanajuato can be seen, and on top of it, one of the domes of the Astronomical Observatory.

This Observatory has been an important center for science outreach since 2006 [2]. Some of the most important activities held at the Observatory are:

- Public talks on Astronomy and other scientific topics.

- Daily astronomical observations, open to a wide public, for free.

- Day time visits of school groups (9-12 years old children), to whom we propose a short astronomy talk. They discover the use of the telescope and make (safe) observations of the Sun and the Moon (see Fig. 2).

- Astronomy courses for teachers are organized every year (see Fig. 3). These courses last a minimum of 30 hours and the program includes the follwoing topics: the celestial sphere, the Solar System, the Stars and the Galaxies. A workshop for using the telescopes and brief introductions to Astrobiology and Cosmology are given too. 


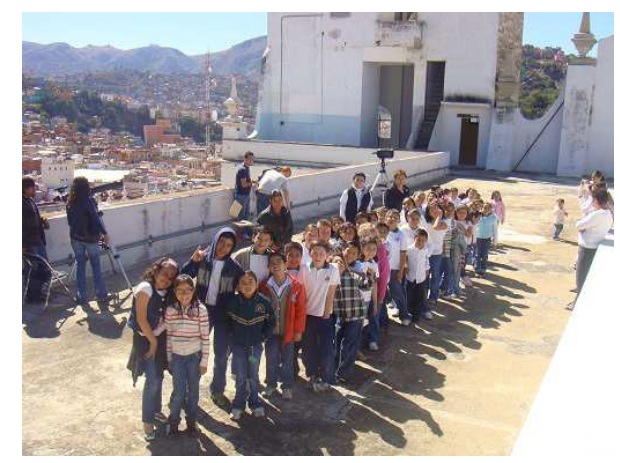

Figure 2. A primary school group visiting the Observatory for day-time observations and science activities.

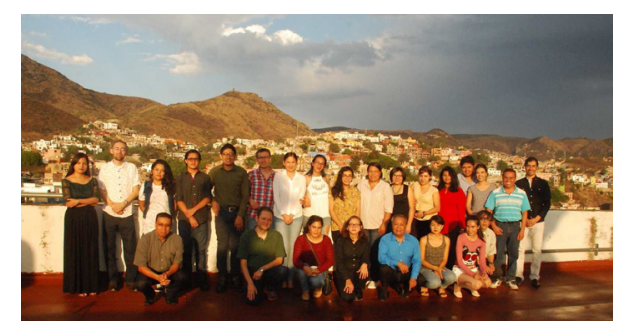

Figure 3. Participants of the 8th Astronomy Course for teachers, held at the Astronomical Observatory, on 2017.

\subsection{Astronomy and Sociology: the contact point}

Concerning the socio-economic situation of the State of Guanajuato, the region has shown dynamic industry development in the last years, but this is very much concentrated along a clear SE-NW axis, including the large cities of the region known as Bajio (the "lower-lands", e.g. León, Salamanca and Celaya). However, most of the NE and SW regions remains rather poor and, for decades, has shown a high migration rate to the USA. It is in this context that a group of sociologists, now part of our team (FMG, PCR, EJS), had developed a series of projects in different zones of the State. Their goal is to improve global life conditions of the local population through well defined activities within a frame of sustainable development. These projects include training of local people in the management of activities like ecotourism, gastronomy, and fair trading of handcrafts and traditional farm products.

(see Sect. 2).

It was during the International Year of Astronomy (IYA09) that the two teams, astronomers and sociologists, got in contact and started a project altogether. This happened after a specific activity: the Mexican Stargazing Night of 2009. Forty sites across the country organized their own Noche de las Estrellas. The one happening in Guanajuato had several thousands of visitors at the archaeological site of Plazuelas, in the south of the State. Seeing the success of these popular stargazing nights, we (astronomers and sociologists) found a common goal: taking these science activities to specific towns in the countryside. This has shown to be an efficient attractor of crowds who are intersted in Astronomy to visit those towns where tourism projects were under development, which were initially poorly known. 
The astronomical branch of the project is driven by professional scientists but many undergraduate and graduate students play a very important role to enable us to reach our goals. Some of the first results of this collaboration were reported in [2].

\section{Preserving the cultural heritage in Guanajuato: the synergy of Sociology and Astronomy}

One of the most important tasks of a public university is the interaction with vulnerable social groups with the purpose of improving their social environment. This can be done through exchanging knowledge and experience, so that a sustainable development approach can be applied. This guideline us to propose, in 2008, a project of Certification on Sustainable Rural Tourism, under the label of $L a$ Universidad en tu Comunidad (The University in your community), with the aim (among others) of preserving and spreading the cultural heritage of State of Guanajuato. The first results of this project are published in [3]. In practice, one of our main goals was to open up new job opportunities on NW villages of the State, many of them difficult to access because of the steep mountains where they are embedded. Several of the regions chosen for this project enjoy natural and/or cultural treasures: archaeological sites, cave paintings, local gastronomy, handcrafts, etc.

After concluding the training in the first villages, sociologists FMG and PCR attended the stargaze nights organized by the astronomers of UG (2009), and realized that combining our efforts could make the tourism projects better known throughout neighbor cities: broadcasting an Astronomical stargazing night, organized at a selected village, could host several hundreds of visitors. This strategy has shown to be a powerful tool to make the full project more visible. In every single case we obtained a very positive reaction of local mayors, who supported the organization and provided transportation and good locations to carry out the activities, which include public talks, science games and, after night fall, astronomical observations. The large number of people taking part in our common activities, both tourists and local people (typically several hundreds), shows how successful this collaboration has been so far.

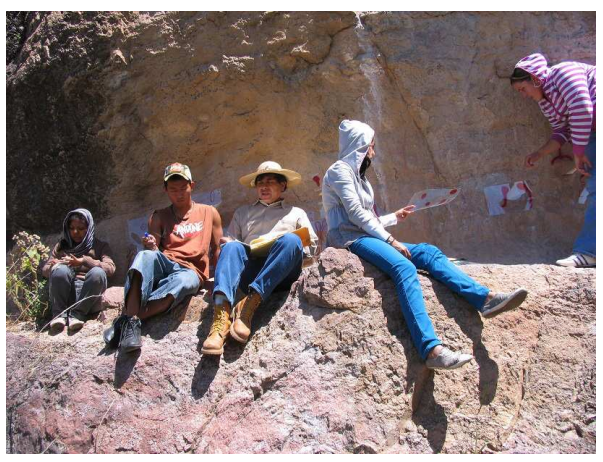

Figure 4. Site el Indio (Sierra del Cubo) in San Felipe, where cave paintings have been found and are currently under study.

Several sites deserve further attention but here we name only two of them. The first is located near the city of San Felipe, in the north of the State, where a mural at the cave "The Indian", and a 
nice landscape (Sierra del Cubo), constitutes a reference within our project (see Fig. 4). Another site at the municipality of Victoria, in the NE of the State, has been very successful case too. Here, the quality of the night sky, an interesting archaeological site, the cave paintings found at Cerro los Remedios, and an exceptional landscape, make of this spot a very touristic one, having a positive impact on job opportunities for local people.

\section{Astronomy goes to the countryside}

This outreach program is part of the larger project La Universidad en tu Comunidad (see Sect. 2). This activity has the main goal to spread some Astronomy knowledge among a wide public, mainly young children. We take advantage of the astonishing objects and phenomena of the sky in order to promote some basic scientific education. Given the (generally) low level of previous knowledge about sciences among our public, special care is taken to adapt the language of complex physical concepts into more simple words. The stargazing is scheduled during the low-rain season, i.e. between November and May.

\subsection{Playful science activities}

We apply different didactic tools, such as talks, playful games, paper-folding workshops, and projections under a portable planetarium. The games are normally organized by sitting people around large tables (children and their parents), each table devoted to different games and workshops. The activities explore topics like: the Solar System, well-known astronomical objects, the Milky Way, Science History, Environment, Maths, Biology, etc. All these activities begin early in the evening, a few hours before sunset (see Fig. 5).

We have produced (and collected) several science games with the aim to promote a community among friends and families, in opposition to the typical (and very individual) use of cellphones and electronic toys. Additionally we use these games as didactic material to introduce the public to some interesting science topics. Here we list a few examples of such activities:

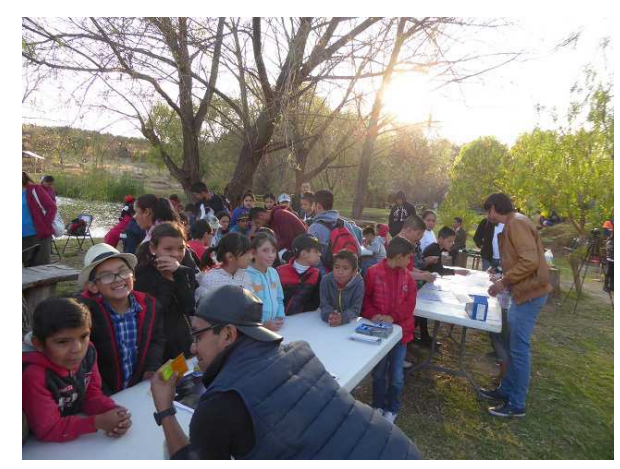

Figure 5. Playful Science Games during an event carried out on 2017, at Comunidad de las Mariposas (the Village of Butterflies), in the north of Guanajuato. 
- Our "visual memory" cards-game is aimed for very young children. The topics drawn on the cards are the planets and other astronomical objects, as well as known scientists, archaeological sites in Mexico, etc.

- Dominoes, using cards with the same themes as above, are for children above five years old.

- We modified the classical "bingo", so that the icons are related to the same topics mentioned before. The moderator says -loudly- a phrase describing the object to select, instead of the name of the object itself; for instance: "The nearest star from planet Earth", is said for players to mark the Sun icon, and so on. The aim, as in classical bingo, is to fill the bingo-card before the other participants.

- Other games are geared to secondary school students and above. One of them deals with Science History, and is popular among undergraduate students, teachers and professionals. It is a classic "Who am I" game. We produce cards for about a hundred scientists, from different areas of knowledge, each one with ten tips about their personal life and scientific achievements. The game covers all epochs of history, from ancient Greeks, to XX century famous scientists, like Marie Curie, Albert Einstein and Carl Sagan. The moderator reads one tip and the players try to guess who is he/she.

The scientific games are very popular during our events. Furthermore, when sky conditions are not good for sky observations, the games become the chief activity of the event. The goal of the games is that the people always win a symbolic prize (astronomical posters, calendars, etc.) in addition to some scientific knowledge.

\subsection{Telescope time!}

As night falls we drive the people to the main activity: the observation of the sky by using our telescopes. We tipically mount up to ten instruments going from 4 to 11 inches of aperture. This activity is driven by 2-3 professional astronomers, assisted by a dozen of students, either undergraduate or MSc-PhD students. We also use green lasers, sky-maps and a few tablets to draw the constellations and describe the celestial sphere movement (see Fig. 6).

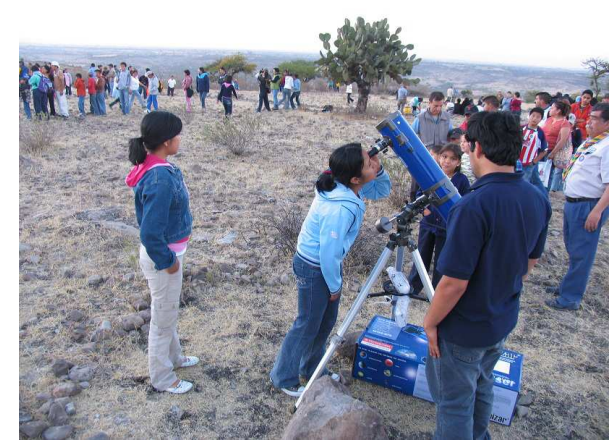

Figure 6. Program "Astronomy goes to your Community", near the town of Dolores-Hidalgo, just before stargazing began. The event took place on top of the "Cerro del Gusano", where some archaeological vestiges remain unexplored. 
The observations usually start 30 minutes or one hour after sunset (it depends of the presence of bright objects, like the Moon or planets). We share the time between live talks, in the dark, while pointing to celestial objects with the help of sky lasers. We comment on the main features of the observed objects, we "draw" the constellations and tell some of the mythology tales associated with them.

Full events last around 6-8 hours in total. The attendance oscillates between several hundreds up to a few thousand participants. Most of the people are local from the host village, but includes as well neighboring communities, and school groups from larger cities of the same region. Very importantly, the logistics (advertisement, security, toilets, water, food, transportation, electricity, etc.) are always provided by the municipality of the same community.

\section{Other stargazing in Guanajuato}

In addition to the stargazing organized at remote villages of the State of Guanajuato, we produce similar events in cities like Guanajuato and Leon, with slightly different goals. The activities are supported by the local Institute of Culture and by Universidad de Guanajuato. The goals are similar to those described above, but the public, logistics and results are very different.

One of our most interesting programs began in 2013 and was named: Mi barrio modelo (My model borough). It consists of stargazing nights and the same activities described above, but now devoted specifically to trouble districts (i.e. not the safest in town) with a high rate of young people leaving too early their studies. For this reason a special logistics backed our work. All activities are conducted by graduate students of our Astronomy MSc and PhD programs, under supervision of professional astronomers and always reported a very positive reaction among participants.

In parallel to the astronomical observations, we developed some games and workshops suited to a young public. A very popular one is the "Planets icosahedron", where children cut, assemble and glue, a mosaic of pictures until getting a 3D representation of a planet surface (see Fig. 7). Another low cost activity that we have developed, is the building of mobiles showing different orbits, like "Earth-Moon", and "Mars and its satellites". Other games are played, like those mentioned in Sect. 3.1. Depending on the local facilities in situ, we display up to six (8"-aperture) telescopes and some tablets and all the necessary material for workshops and talks. We normally take advantage of sport fields for the observations, while games and talks use to be organized within closed sport complex.

\section{Conclusions}

During already nine years, starting since IYA2009, the collaboration between astronomers and sociologists of Universidad de Guanajuato has shown to be very fruitful, having a very positive impact on several aspects like preservation of cultural heritage and improvement of education and socioeconomic conditions of selected villages in the State of Guanajuato. A third branch of this project concerning environment preservation is currently developed, with the collaboration of biologists and environment engineers of the same university. These results will be described in forthcoming papers.

The present project is nowadays very well known across the State making that several communities must go through a "waiting list" before getting scheduled within our program. 


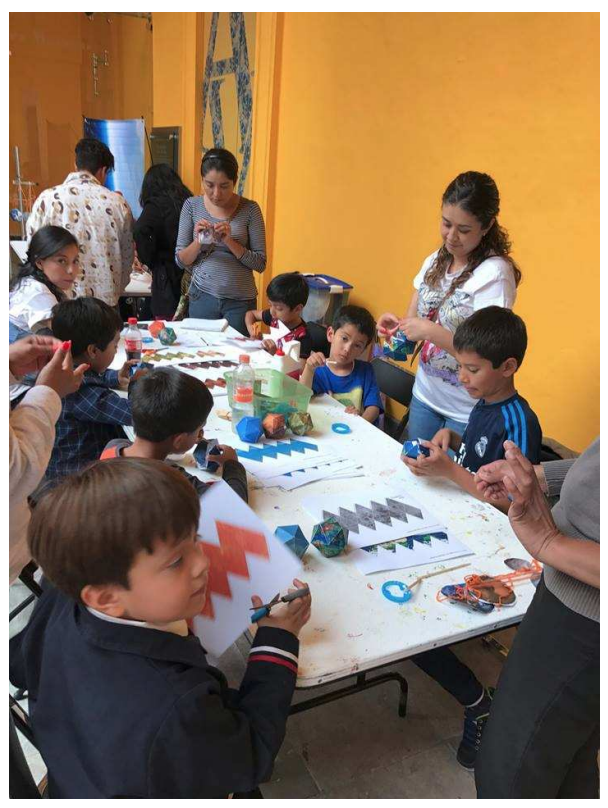

Figure 7. Children attending the workshop "Planets Icosahedron"

\section{References}

[1] Bravo-Alfaro, H. 2007, IAU Special Session \#5, Astronomy for the Developing World. 26th meeting of the IAU. J.B. Hearnshaw and P. Martinez, Eds. Cambridge University Press, 2007, pp.59

[2] Bravo-Alfaro, H.; Caretta, C. A.; Brito, E. M. S.; Campos, P.; Macias, F. 2015, in Highlights of Astronomy, International Astronomical Union, Volume 16, pp. 648

[3] Macias Gloria, F., Campos Rodríguez, P., et al. Eds. Cultura, Patrimonio, ciencia, tecnología y desarrollo. Universidad de Guanajuato, Mexico, 2017. 